\title{
Actividad física y ejercicio asociados con ansiedad y depresión clínica durante la pandemia por COVID-19
}

\section{Physical activity and exercise associated with anxiety and clinical depression during the COVID-19 pandemic}

\author{
Gloria Vargas-Sánchez*, Isabel Ma. Aguilar-Ortiz y Arturo Goiz-Joachin \\ Investigación en Ciencias del Deporte, Universidad del Fútbol y Ciencias del Deporte, Club de Fútbol Pachuca, Pachuca, Hidalgo, México
}

\begin{abstract}
Resumen
Introducción: La actividad física se considera una de las fuerzas más poderosas para mantener la salud. La COVID-19 es la enfermedad infecciosa causada por el nuevo coronavirus 2 del síndrome respiratorio agudo grave y la causa de la pandemia actual. Objetivo: Analizar la asociación entre actividad física, ejercicio, y la presencia de ansiedad y/o depresión clínica durante la pandemia. Material y métodos: Mediante encuesta descriptiva, se estudió una muestra de 504 sujetos. Variables identificadas: actividad física, ejercicio, depresión, ansiedad, edad y sexo. Se realizó análisis de $\chi^{2}$ para estudiar la relación entre las variables, y análisis de la varianza para grupos de edad. Resultados: Se encontró ansiedad clínica en el $67.26 \%$ de la muestra, prevalente en el sexo femenino (46.62\%), con una diferencia significativa al comparar el grupo que no realiza actividad física con el que sí lo hace $(p=0.009)$. Se halló depresión clínica en $28.57 \%$ de la muestra, prevalente en el sexo femenino $(32.12 \%)$, con una diferencia significativa al comparar el grupo que no realiza actividad física con el que sí lo hace ( $p<0.010$ y $p<0.015$ respectivamente). El grupo más significativo fue el de 18 a 35 años de edad ( $p<$ 0.005). Conclusiones: Se encontró menor asociación en estados de ansiedad y depresión clínica en aquellas personas que realizan actividad física y ejercicio, siendo significativamente mayor en el sexo femenino.
\end{abstract}

Palabras clave: Actividad física. Ejercicio. Pandemia. COVID-19. Ansiedad. Depresión.

\begin{abstract}
Background: Physical activity is considered one of the most powerful forces for maintaining health1. COVID-19 is the infectious disease caused by a new coronavirus and the cause of the current Pandemic. Objective. Analyze the association between physical activity, exercise, the presence of anxiety and/or clinical depression during the pandemic. Material and methods. By descriptive survey; $n=504$ was studied, the identified variables: physical activity, exercise, depression, anxiety, age and sex. $\chi^{2}$ analysis was performed for the relationship between the variables, analysis of variance for age groups. Results. Clinical anxiety was found in $67.26 \%$ of the population, prevalent in the female sex (46.62\%), with a significant difference when comparing the population that did not perform physical activity $(p=0.009)$. Clinical depression was found in $28.57 \%$ of the population and it was prevalent in the female sex $(32.12 \%)$ with a significant difference when comparing the population that does not perform physical activity $(p<0.010$ and $p<0.015$ respectively). The most significant group was 18 to 35 years of age $(p<0.005)$. Conclusions. A lower association was found in states of anxiety and clinical depression in those people who perform physical activity and exercise, being significantly higher in the female sex.
\end{abstract}

Key words: Physical activity. Exercise. Pandemic. Covid-19. Anxiety. Depression.

\footnotetext{
Correspondencia:

Fecha de recepción: 07-09-2020

*Gloria Vargas-Sánchez

E-mail: gloria.vargas@tuzos.com.mx

Fecha de aceptación: 07-10-2020

DOI: 10.24875/RHJM.20000108

1405-9622/@ 2020 Sociedad Médico-Quirúrgica del Hospital Juárez de México, A.C. Publicado por Permanyer. Este es un artículo open access bajo la licencia CC BY-NC-ND (http://creativecommons.org/licenses/by-nc-nd/4.0/).
} 


\section{Introducción}

La actividad física tiene efectos inmediatos sobre el funcionamiento del sistema inmunitario y la inflamación ${ }^{1}$. Similar a tomar un medicamento diariamente, las personas pueden reducir su riesgo de infecciones virales graves y el riesgo de múltiples enfermedades crónicas simplemente intentando cumplir la recomendación de actividad física de 150 minutos a la semana (actividad física moderada aeróbica), lo que podría cumplirse con 30 minutos diarios de actividad física en casa o dando un paseo corto, según estipuló la Organización Mundial de la Salud (OMS) en 2019. Por otra parte, estar físicamente activo tiene importantes beneficios para la salud mental, por lo que alentar a las personas a estar activas podría ayudar a muchos a sobrellevar la depresión, el estrés continuo y evitar enfermedades psicológicas. De acuerdo con Basso, et al. cada sesión de actividad física reduce los síntomas de ansiedad y depresión, por lo que estar activo todos los días puede ser un antídoto parcial para el estrés ${ }^{2}$. Para las personas que ya sienten depresión, estrés y angustia, estar activo es tan efectivo como los medicamentos y la psicoterapia ${ }^{3}$.

El nuevo coronavirus y la enfermedad por coronavirus 2019 (COVID-19) eran desconocidos antes de que estallara el brote en Wuhan (China) en diciembre de $2019^{4}$. La autoridad sanitaria a nivel mundial, la OMS, ha pedido a la población que ante la pandemia exista confinamiento y estar cuando menos a dos metros de distancia (directriz a la que se adhiere la Secretaría de Salud del Gobierno de México) ${ }^{5}$, para ayudar a prevenir su propagación. En México, el 23 de marzo de 2020 las autoridades sanitarias emitieron el anuncio de la campaña: "Quédate en casa» como una medida preventiva durante la fase 2 para evitar una propagación de contagio masivo ${ }^{5}$.

Ante la escasez de estudios que demuestren asociación entre la actividad física, el ejercicio, la presencia de ansiedad y la depresión, nuestro estudio analiza esta relación entre los factores asociados en la fase de confinamiento. Se desconoce si el efecto es mayor en hombres o en mujeres. De acuerdo con una investigación realizada en China por Wang, et al. en la población general, se encontró que existe un aumento en la depresión conforme pasan los días de la pandemia durante la etapa inicial de la COVID-196. Otro estudio interesante es el realizado por James, et al., en el cual se ha demostrado que la ansiedad y el confinamiento mejoran con la práctica de actividad física?

\section{Métodos}

Nuestro estudio es de tipo observacional, descriptivo, exploratorio y transversal. Se aplicó una encuesta digital en la que participaron 509 personas (330 mujeres y 174 hombres). La investigación fue aprobada por un comité de ética de la Universidad de Fútbol y Ciencias del Deporte. La encuesta fue aplicada durante dos semanas, a partir de la segunda semana en que fue declarada la fase 2 en México ( 25 de marzo de 2020), en las regiones centro, noreste, noroeste, sureste y occidente. Una vez recolectados los datos fueron codificados y posteriormente analizados con el programa estadístico SPSS versión 25.

Con la información recopilada se realizó un análisis de asociación de $\chi^{2}$ a dos colas, para identificar la relación entre las variables independientes; actividad física, ejercicio, sexo, edad, ansiedad y depresión. En nivel de significancia estadística se trabajó con una $p<0.05$. A partir del análisis de ANOVA de una vía se identificaron los efectos de las variables grupo de edad, sexo, depresión y ansiedad.

\section{Resultados}

Los datos indicaron estadísticamente la presencia de síntomas y signos de ansiedad en la muestra estudiada. Se encontró ansiedad clínica en el $67.26 \%$ de la muestra $(n=339)$. Fue prevalente en el sexo femenino, mayor en mujeres que en hombres, el $46.62 \%$ del total de la muestra ( $n=235)$ vs. el $20.63 \%(n=104)$, con una diferencia significativa al comparar la población que realiza ejercicio con aquella que no realiza ejercicio, demostrando una significancia $(p=0.009)$. Se encontró depresión clínica en el $28.57 \%$ de la muestra, con prevalencia en el sexo femenino (32.12\%), con una diferencia significativa al comparar la población que no realiza actividad física $(p<0.010$ y $p<0.015$ respectivamente).

En la tabla 1 podemos apreciar las cifras de ansiedad y su relación con el ejercicio y el sexo.

En la tabla 2 podemos apreciar las cifras de depresión y su relación con el ejercicio y el sexo.

En la figura 1 podemos apreciar que el $46 \%$ de mujeres tiene depresión.

En la figura 2 podemos apreciar que el $21 \%$ de mujeres tiene depresión.

Encontramos resultados significativos para los grupos de edad, con F: 9.737, p = 0.000, y sexo, con F: $5.180, p=0.023$. Se observó una clara diferencia entre los grupos de edad, de 18 a 35 años, 36 a 50 años y 
mayores de 50 años $(p<0.05)$. Los resultados para depresión con esta variable fueron significativos. El grupo de edad en el que se encontró mayor significancia para sintomatología depresiva fue el de 18 a 35 años de edad ( $\mathrm{F}:$ 4.902, $\mathrm{p}=0.027)$.

En las figuras 3 y 4 podemos observar medidas marginales de Ansiedad y Depresión por grupo de edad.

\section{Discusión}

En nuestro estudio se encontró una prevalencia alta de ansiedad y depresión durante la fase 2 de la crisis sanitaria por COVID-19; se encontró asociación estadísticamente significativa con el sexo, la edad y la actividad física. Las mujeres son mayormente afectadas. Los sujetos que declararon haber realizado actividad física y ejercicio durante esta fase de confinamiento presentaron menores niveles de ansiedad y depresión.

En México antes de la pandemia por COVID-19 se realizaron numerosas investigaciones para estudiar ansiedad y depresión, pero ninguna en condiciones de confinamiento y su relación con la actividad física y ejercicio. Por ejemplo, Berenzon, et al. realizaron una breve revisión de investigaciones que permiten ubicar la depresión como un problema de salud pública en México de importantes costos, refieren que requiere tomar acciones para prevenir y tratar, mediante un proceso continuo de evaluación de los programas y de las políticas públicas ${ }^{8}$. Por su parte, Zhang, et al. realizaron un estudio longitudinal con estudiantes universitarios para analizar los problemas de salud mental y el ejercicio como un efecto de mitigación ${ }^{9}$. Encontraron que la COVID-19 afectó negativamente las emociones y la calidad del sueño, pero que una posible estrategia de mitigación es realizar una cantidad suficiente de actividad física diariamente y dormir bien ${ }^{10}$.

Por su parte, Seshadri realizó un estudio en India que indica que en casos ya diagnosticados de trastorno de ansiedad y depresión es probable que se provoque una exacerbación de los síntomas, debido a que la cuarentena es una situación estresante que aumenta la morbilidad psiquiátrica por medio de diferentes mecanismos ${ }^{10}$. Estos dos hallazgos pueden justificar en parte los niveles de ansiedad y depresión que hemos encontrado en el caso de México durante nuestro estudio, que se sitúan por arriba de los hallados en otros países. Por ejemplo, Wang, et al. realizaron un estudio en China de tipo longitudinal durante dos fases en las cuales se evaluó el impacto de la COVID-19 en la salud mental de población general ${ }^{6}$. Durante la evaluación inicial, se observó el $8.1,28.8$ y $16.5 \%$, de estrés
Tabla 1. Cifras de ansiedad y su relación con ejercicio y sexo

\begin{tabular}{|c|c|c|c|}
\hline \multirow{2}{*}{ Sexo } & \multicolumn{2}{|c|}{ Ejercicio } & \multirow{2}{*}{ Total } \\
\cline { 2 - 3 } & No & Sí & \\
\hline Femenino & & & \\
Ansiedad & & & \\
No & 37 & 58 & 95 \\
Sí & 108 & 127 & 235 \\
Total & 145 & 185 & 330 \\
\hline Masculino & & & \\
Ansiedad & & & \\
No & 26 & 44 & 70 \\
Sí & 61 & 43 & 104 \\
Total & 87 & 87 & 174 \\
\hline Total & & & \\
Ansiedad & & & \\
No & 63 & 102 & 165 \\
Sí & 169 & 170 & 339 \\
Total & 232 & 272 & 504 \\
\hline
\end{tabular}

Tabla 2. Cifras de depresión y su relación con ejercicio y sexo

\begin{tabular}{|c|c|c|c|}
\hline \multirow{2}{*}{ Sexo } & \multicolumn{2}{|c|}{ Ejercicio } & \multirow{2}{*}{ Total } \\
\cline { 2 - 3 } & No & Sí & \\
\hline Femenino & & & \\
Depresión & & & \\
No & 91 & 133 & 224 \\
Sí & 54 & 52 & 106 \\
Total & 145 & 185 & 330 \\
\hline Masculino & & & \\
Depresión & & & \\
No & 63 & 73 & 137 \\
Sí & 25 & 13 & 37 \\
Total & 88 & 86 & 174 \\
\hline Total & & & \\
Depresión & & & \\
No & 155 & 205 & 360 \\
Sín & 79 & 65 & 144 \\
Total & 234 & 270 & 504 \\
\hline
\end{tabular}

moderado a severo, ansiedad y depresión, respectivamente, y no hubo cambios longitudinales significativos en los niveles de estrés, ansiedad y depresión ( $p$ > 0.05). González, et al. analizaron las consecuencias en la salud mental en España durante la fase inicial de la pandemia de COVID-19, encontrando que el $18.7 \%$ de la muestra reveló una sintomatología depresiva y el $21 \%$ ansiedad, adicionalmente que las mujeres previamente diagnosticadas con COVID-19 tuvieron asociada esa sintomatología ${ }^{11}$. Kapilashrami, a partir de su investigación, ha encontrado evidencia de que esta pandemia debida al COVID-19 parece que fue más dañina para algunos grupos que otros, y que las enfermedades mentales son más comunes en estas minorías ${ }^{12}$. En un 


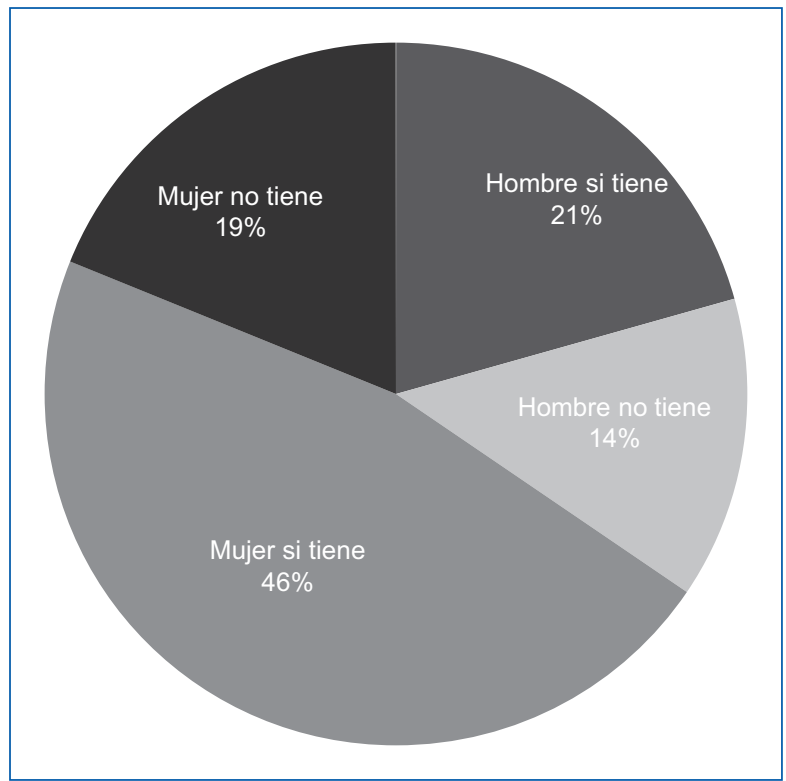

Figura 1. Porcentajes de depresión.

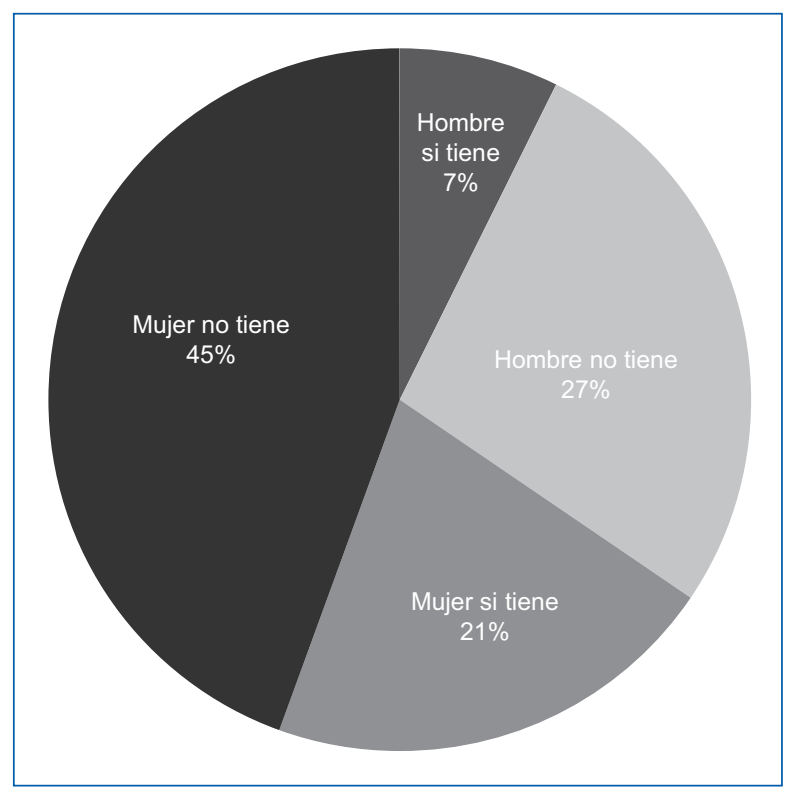

Figura 2. Porcentajes de ansiedad.

metaanálisis realizado con el objetivo de estimar la edad de inicio (EDI) para todos los trastornos de ansiedad, se encontró una EDI media de todos los trastornos de ansiedad de 21.3 años (intervalo de confianza del 95\%: 17.46-25.07 años) $)^{13}$. El trastorno de ansiedad generalizada comenzó, en promedio, entre 21.1 y 34.9 años. El metaanálisis no reveló diferencias en la EDI entre sexos. Un diseño de estudio prospectivo y un

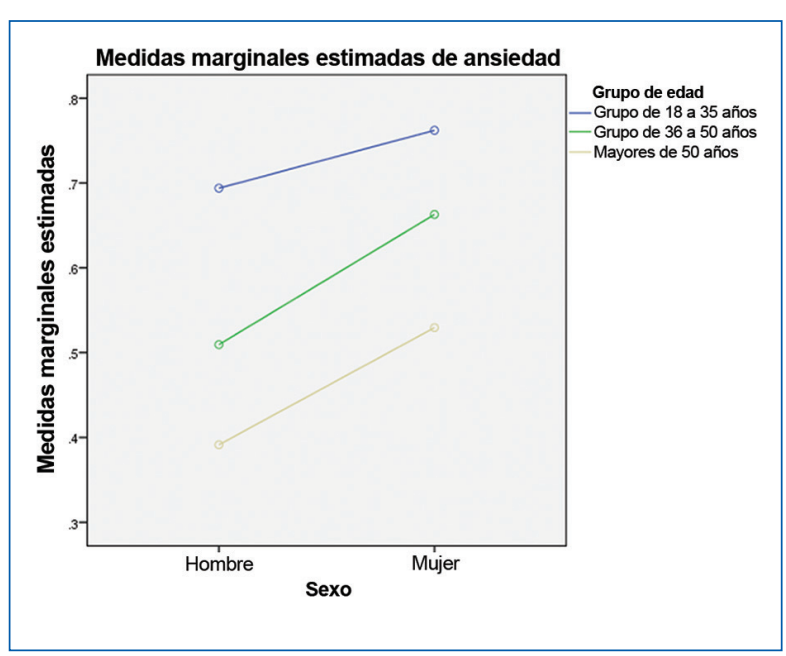

Figura 3. Medidas marginales de ansiedad por grupo de edad.

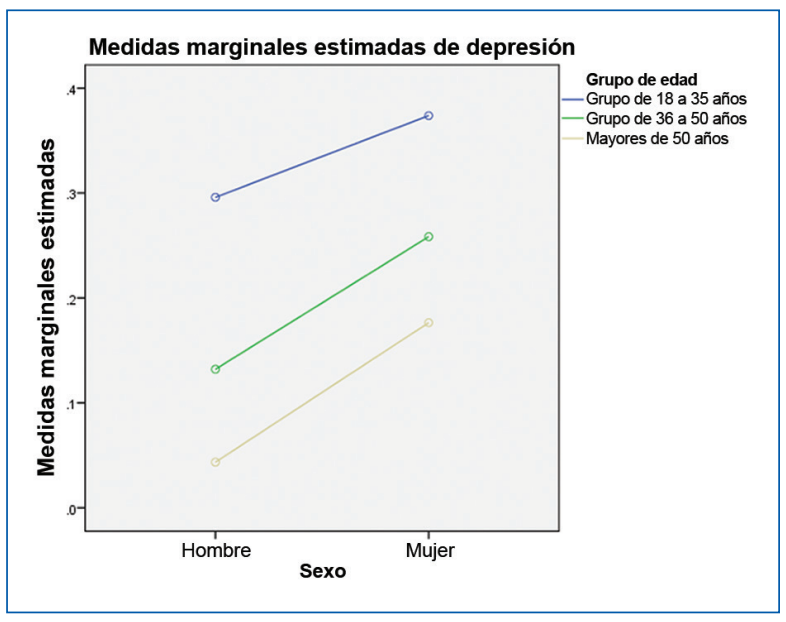

Figura 4. Medidas marginales de depresión por grupo de edad.

mayor nivel de desarrollo del país de estudio se asociaron con una EDI temprana ${ }^{13}$. En la investigación que se presenta en este documento se ha encontrado que el sexo influye en el trastorno de ansiedad y depresión, encontrado que las mujeres son las que presentan el mayor porcentaje, las mujeres que realizaron actividad física redujeron los síntomas de ansiedad y depresión respecto a quienes no realizan actividad física ${ }^{14}$.

\section{Conclusiones}

La actividad física y el ejercicio son elementos importantes que contribuyen en la disminución de síntomas y 
signos de ansiedad y depresión durante la pandemia por COVID-19. Encontramos evidencia estadísticamente significativa de que el sexo femenino es el más afectado ante situaciones de confinamiento. Cabe resaltar que el porcentaje de sujetos afectados por ansiedad y depresión que hemos encontrado en este estudio supera los números referidos y hallazgos en otros países.

\section{Agradecimientos}

Agradecimiento especial a la Universidad del Fútbol y Ciencias del Deporte, al Comité de Investigación y en especial a la Rectora de la Universidad, por su invaluable apoyo.

\section{Conflicto de intereses}

Los autores declaran no tener conflicto de intereses alguno.

\section{Financiamiento}

Los autores no recibieron patrocinio para llevar a cabo este artículo.

\section{Responsabilidades éticas}

Protección de personas y animales. Los autores declaran que para esta investigación no se han realizado experimentos en seres humanos ni en animales.

Confidencialidad de los datos. Los autores declaran que han seguido los protocolos de su centro de trabajo sobre la publicación de datos de pacientes.
Derecho a la privacidad y consentimiento informado. Los autores declaran que en este artículo no aparecen datos de pacientes.

\section{Bibliografía}

1. Brosse AL, Sheets ES, Lett HS, Blumenthal JA. Exercise and the treatment of clinical depression in adults. Sports Med. 2002;32:741-60.

2. Hojman $P$. Exercise protects from cancer through regulation of immune function and inflammation. Biochem Soc Trans. 2017;45(4):905-11.

3. Arrivillaga M, Cortés C, Goicochea J, Lozano T. Caracterización de la depresión en jóvenes universitarios. Universitas Psychologica. 2003;1(3):17-25.

4. Coronavirus [Internet]. Organización Mundial de la Salud; 2019 [citado: 8 jun 2020]. Disponible en: https://www.who.int/es/health-topics/coronavirus

5. Todo sobre el COVID-19 [Internet]. México: Gobierno de México, Secretaría de Salud; 2020 [citado: 8 jun 2020]. Disponible en: https://coronavirus.gob. $\mathrm{mx} /$

6. Wang C, Pan R, Wan X, et al. A longitudinal study on the mental health of general population during the COVID-19 epidemic in China. Brain Behav Immun. 2020;87:40-8.

7. Sallis J, Pratt M. La actividad física puede ser útil en la pandemia de coronavirus. Global Observatory for Physical Activity [Internet] [consultado: 02 de abril de 2020]. Disponible en: http://www.incap.int/index.php/ es/covid-19/487-la-actividad-fisica-puede-ser-util-en-la-pandemia-de-coronavirus-gopa/file

8. Berenzon S, Lara MA, Robles R, Medina-Mora ME. Depresión: estado del conocimiento y la necesidad de políticas públicas y planes de acción en México. Salud Publica Mex. 2013;55(1):74-80.

9. Zhang $\mathrm{Y}$, Zhang $\mathrm{H}, \mathrm{Ma} X, \mathrm{Di}, \mathrm{Q}$. Mental health problems during the COVID19 pandemics and the mitigation effects of exercise: A longitudinal study of college students in China. Int J Environ Res Public Health. 2020;17:3722.

10. Chatterjee SS, Barikar C M, Mukherjee A. Impact of COVID-19 pandemic on pre-existing mental health problems. Asian $\mathrm{J}$ Psychiatr. 2020;51:102071.

11. González-Sanguino C, Ausín B, Castellanos MÁ, Saiz J, López-Gómez A, Ugidos $\mathrm{C}$, et al. Mental health consequences during the initial stage of the 2020 Coronavirus pandemic (COVID-19) in Spain. Brain Behav Immun. 2020;87:172-6.

12. Kapilashrami A, Bhui K. Mental Health \& COVID-19: is the virus racist? Br J Psychiatry. 2020;217(2):405-7.

13. Lijster JM, Dierckx B, Utens EM, Verhulst FC, Zieldorff C, Dieleman GC et al. The age of onset of anxiety disorders. Can J Psychiatry. 2017;62(4):237-46.

14. Medina JL, DeBoer LB, Davis ML, Rosenfield D, Powers MB, Otto MW, et al. Gender moderates the effect of exercise on anxiety sensitivity. Ment Health Phys Act. 2014;7(3):147-51. 